\title{
HPV E6 Protein Associated SOX21-AS1 Promotes YAP/TAZ Activation by Attenuating YAP1 Ubiquitination in Human Cervical Cancer
}

Qin Liu

First Peoples Hospital of Kunshan

Zhiwei Zhu

First Peoples Hospital of Kunshan

Senlin Lian

Nanjing Medical University

Lixia Zhu

First Peoples Hospital of Kunshan

Jiahui Pan

Jilin University

\section{Song $\mathrm{Xu}$}

First Peoples Hospital of Kunshan

\section{Xuegang Zhang}

First Peoples Hospital of Kunshan

Juping Zhang

First Peoples Hospital of Kunshan

Huizi Xu

First Peoples Hospital of Kunshan

\section{Yun Chen}

Nanjing Medical University

\section{Guoqing Wang}

Jilin University

Jiang Runqiu ( $\sim$ Jiangrq@njmu.edu.cn )

Liver transplantation center, Nanjing Medical University https://orcid.org/0000-0002-6995-9557

\section{Primary research}

Keywords: Cervical Cancer, YAP1, IncRNA, and E6

Posted Date: August 12th, 2020

DOI: https://doi.org/10.21203/rs.3.rs-55342/v1 
License: (c) (i) This work is licensed under a Creative Commons Attribution 4.0 International License. Read Full License 


\section{HPV E6 protein associated SOX21-AS1 promotes YAP/TAZ activation by attenuating}

\section{YAP1 ubiquitination in human Cervical Cancer}

Running title: E6 derived SOX21-AS1 promote CCs by Hippo signaling

Key words: Cervical Cancer; YAP1; lncRNA; and E6

Liu Qin ${ }^{1 \#} ;$ Zhu Zhiwei ${ }^{1 \#}$; Lian Senlin² ${ }^{2 \#}$ Zhu Lixia ${ }^{1}$; Pan Jiahui ${ }^{4} ;$ Xu Song ${ }^{3}$; Zhang Xuegang ${ }^{1}$;

Zhang Juping ${ }^{1}$; Xu Huizi ${ }^{1}$; Chen Yun²; Wang Guoqing ${ }^{4 *}$ and Jiang Runqiu ${ }^{5,6^{*}}$

1. Department of Gynaecology, Kunshan First People's Hospital, Affiliated to Jiangsu University, Kunshan, 215300, People's Republic of China.

2. Department of Immunology, Nanjing Medical University, Nanjing, 210000, People's Republic of China.

3. Department of Pathology, Kunshan First People's Hospital, Affiliated to Jiangsu University, Kunshan, 215300, People's Republic of China.

3. Department of Pathogenobiology, The Key Laboratory of Zoonosis, Chinese Ministry of Education, College of Basic Medicine, Jilin University, Changchun, 130021, China.

4. Department of Hepatobiliary Surgery, The Affiliated Drum Tower Hospital of Nanjing University Medical School, Nanjing, Jiangsu, China.210093. People's Republic of China

5. Medical School of Nanjing University, Nanjing, Jiangsu, China. 210093. People's Republic of China

\section{Corresponding Author:}

Jiang Runqiu, jiangrq@ nju.edu.cn. 22 Hankou Rd. Nanjing, Jiangsu, China. (86)-25-83593186. or Wang Guoqing, qing@jlu.edu.cn. 126 Xinmin Ave.Changchun, Jilin, China. (86)- 0431-85619463 Word count:4880 words. 


\begin{abstract}
Background: The human papillomavirus (HPV), especially HPV 16 type infection, is one of the well-known causes for human cervical cancer, we sought to investigate HPV 16 regulating lncRNA and its role in cervical carcinogenesis.
\end{abstract}

Methods: The potential HPV16 related lncRNAs were screened by using bioinfomatic analysis. The relationship between SOX21-AS1 and clinical features was studied by using clinical samples. Its oncogenic roles were explored in vitro.

Results: A HPV 16 infection-specific lncRNA, SOX21-AS1 was spotted by screening the Cancer Genome Atlas TCGA database, and its expression was seriously related to Hippo signaling pathway by using Gene Set Enrichment Analysis. Total 40 cases patients suffered from cervical carcinomas (CCs) were involved, we found that SOX21-AS1 was over-expressed in those CCs patients who were positive for HPV 16 infection compared to those who were negative. We also found that SOX21-AS1 expression was positively related to E6 protein but not E7 expression, which is a carcinogenic protein of HPV 16. Also, YAP1/TAZ/TEAD complex can increase SOX21-AS1 transcription by binding to its promoter region. SOX21-AS1 can promote cell proliferation and invasion in CCs cell lines in a YAP1 activation-dependent manner. We found that SOX21-AS1 can promote YAP1 activation by preventing YAP1 phosphorylation at s127 residue and further preventing its degradation by binding to 14-3-3.

Conclusion: In conclusion, SOX21-AS1 is an HPV16 specific lncRNA which can promote tumor growth and metastasis by targeting the Hippo signaling pathway.

Key words: Cervical Cancer; YAP1; lncRNA; and E6 


\section{Introduction}

The human papillomavirus (HPV) infection is the world's most prevalent sexually transmitted disease, infecting about 75 percent of sexually active women [1]. Human HPV infects epidermal or mucosal epithelial cells and can cause benign or malignant tumors [2]. Certain types of HPV, such as HPV-16, -18, -31 and -45 , are considered high-risk or carcinogenic [3]. In high-risk groups, HPV16 and -18 can cause $90 \%$ of cervical cancer [3] and $20 \%$ of oral cancer [2].

Oncoproteins E6 and E7 of high-risk HPV have proved to contribute to carcinogenesis by intervening cellular biological processes, especially by targeting the tumor suppressors including p53 and pRb[4, 5]. For example, E6 protein can attenuate p53 function by degradation through combination with E6-associated protein (E6AP)[6], or by suppression CBP-p300, a p53 coactivator [7]. E7 can bind to the pocket domain of Rb protein, which is an essential domain for the tumor suppressive function[6]. E6 and E7 protein were also reported to modify the cellular transcription by binding to the transcriptional factors such as AP-1[8, 9], E2F[10, 11], and TEADs[12], etc, thus to promote cell proliferation, invasion, and inducing immunosuppression, etc. Besides the regulation of E6/E7 on encoded genes, a few groups have focused on the effect of E6/E7 protein on the expression of Long Non-coding RNAs (lncRNAs). For instance, very recently, Barr. et al. reported FAM83H-AS1 was overexpressed in human HPV type 16 positive cervical cancer cell line in an E6 dependent manner, FAM83H-AS1 have pro-cancer characters by enhancing cellular proliferation and anti-apoptosis in a p53 independent way[13]. In 2012, Villota et al. firstly revealed that E6/E7 can down-regulation of the ASncmtRNAs and induces the expression of a new sense transcript named SncmtRNA-2 [14].

In the present study, we found that an HPV type16 infection-related LncRNA, SOX21-AS1, was overexpressed in HPV 16 positive human Cervical Carcinomas (CCs), our study indicated that this lncRNA was not only a E6 protein-related lncRNA, but also seriously related to YAP/TAZ activation. Moreover, its cell proliferation- and invasion- promotion effects and related molecular mechanism were explored.

\section{Materials and Methods}

\section{Patients}

This hospital-based case-control study included 40 CCs patients. All subjects were recruited from the 1st People's Hospital of Kunshan affiliated of Jiangsu University between January 2016 and January 2019. All patients underwent surgery for primary CCs, and subjects with a previous 
history of cancer or chemotherapy were excluded. The cancer-free controls who were from the same geographic area showed no evidence of a genetic relationship to the CCs patients. This study was approved by the Ethics Review Board of the 1st People's Hospital of Kunshan affiliated of Jiangsu University, and all patients provided written informed consent.

\section{Bioinformatics analysis}

RNA sequencing data for The Cancer Genome Atlas (TCGA) Program human cervical $\begin{array}{llll}\text { cancer } & \text { tissue } & \text { were } & \text { downloaded }\end{array}$ http://ibl.mdanderson.org/tanric/_design/basic/download.html. According to the report by Tang et al.[15], we obtained 18 samples of other HPV infection and 55 samples of HPV 16 infection within TGCA_CESC database. R packages including limma, pheatmap were used to find out differentially expressed lncRNAs and generate heatmaps. Analyzed gene sets were downloaded from GSEA (http://software.broadinstitute.org/gsea/index.jsp).

\section{Cell lines and reagent}

Human CCs cell lines including C33A, ME180, SiHa, and CaSKi were purchased from the American Type Culture Collection (ATCC). All cells were cultured in Dulbecco's Modified Eagle's Medium (DMEM) purchased from Gibco (CA, USA) supplemented with 10\% fetal bovine serum (FBS) (Invitrogen, Carlsbad, CA, USA) and maintained in a humidified atmosphere containing $5 \% \mathrm{CO} 2$ at $37^{\circ} \mathrm{C}$. Lysophosphatidic acid (LPA) was purchased from ENZO (BML-LP100-0025). Inhibitor of YAP1/TAZ was purchased from Selleckchem (s8661).

\section{Real-time PCR}

Total RNA in CCs tissues was isolated using TRIzol reagent. The expression of SOX21-AS1 was detected using the syber-green-based real-time PCR. The primers for genes used in the study were as follows: forward primer: GCATGGGATTCTGACTGAG, reverse primer: AGAGAAGAGATCTATCGCT, amplicon size 157bp for SOX21-AS1, and forward primer: TGTGGGCATCAATGGATTTGG, primer: ACACCATGTATTCCGGGTCAAT, amplicon size 110bp for GAPDH.

\section{RNA pull-down, in vitro ubiquitination and Co-IP assay}

The RNA pull-down assay was performed by using Pierce Magnetic RNA-Protein Pull-Down Kit (Thermofisher, CA) according to the instructions from the manufacturer. Briefly, the total RNA was extracted from C33A and CaSki cells, magnetic beads were incubated with Probes for biotin-labeled SOX21-AS1 (Genescript Co., Nanjing, China) (sequence: 
acaggctgatgcatgggattct) and U6 control (sequence: ttggaacgatacagagaagatt).

For ubiquitination detection, whole cell lysates were prepared in a lysis buffer containing 10 $\mathrm{mM}$ N-Ethylmaleimide to inhibit ubiquitin-conjugating enzymes. YAP1 was immunoprecipitated, and its ubiquitination was detected with anti-ubiquitin antibody (1:1000, ab7254) (Abcam, Cambridge, UK).

For detecting the interaction of TAZ with E6 or E7, CaSki were transduced with indicated E6 or E7. Whole cell lysates were prepared and TAZ was immunoprecipitated with TAZ (ab652, Abcam, Cambridge) via a Dynabeads ${ }^{\circledR}$ Protein A Immunoprecipitation Kit (Thermofisher, CA, USA) according to manufacturer's instruction. The presence of EGFR in the immune complex was determined by Western blot using anti-E6 or E7 antibodies.

\section{Chromatin Immunoprecipitation Assay (ChIP)}

Chromatin immunoprecipitation assay assays were performed via a commercially purchased chromatin immunoprecipitation kit (Millipore, Temecula, CA), using anti-TEAD1, anti-TEAD2, anti-TEAD3, anti-TEAD4 and p65 antibodies, and IgG antibody served as a negative control. Cells were first cross-linked for 10 min by adding formaldehyde directly to tissue culture medium to a final concentration of $1 \%$. Cross-linked cells were then washed twice with cold PBS (with protease inhibitors), scraped, pelleted, resuspended in $200 \mu \mathrm{l}$ SDS lysis buffer (1\% SDS, $10 \mathrm{mM}$ EDTA, $50 \mathrm{mM}$ Tris-HCl, pH 8.0), and incubated for $10 \mathrm{~min}$ on ice. The lysates were then sonicated for five cycles of $30 \mathrm{~s}$ each, resting on ice for $1 \mathrm{~min}$ between cycles. After sonication, the samples were centrifuged and the supernatants diluted 10-fold in ChIP dilution buffer with protease inhibitors and precleared with $60 \mu \mathrm{l}$ salmon sperm DNA/protein A Agarose-50\% slurry for $60 \mathrm{~min}$ at $4^{\circ} \mathrm{C}$. Cross-linked chromatin was incubated overnight with $5 \mu \mathrm{g}$ antibodys mentioned above and control IgG in a total volume of $1 \mathrm{ml}$ at $4{ }^{\circ} \mathrm{C}$. Antibody-protein-DNA complexes were isolated by immunoprecipitation with $60 \mu \mathrm{l}$ salmon sperm DNA/protein A. After extensive washing, pellets were eluted by freshly prepared elution buffer (1\% SDS, $0.1 \mathrm{M}$ $\mathrm{NaHCO} 3$ ). Formaldehyde cross-linking was reversed by $5-12-\mathrm{h}$ incubation at $65^{\circ} \mathrm{C}$ after adding $20 \mu \mathrm{l} 5 \mathrm{M} \mathrm{NaCl}$. Samples were purified through PCR purification kit columns (Qiagen, Chatsworth, CA) and used as a template in RT-qPCR. ChIP primers: 5'-CGAGGGGGCGGTGACAGCA-3' and 5'-CCTCGCTAGCTTTGATGCGCAGA- 3' were used to amplify a 200-base pair fragment corresponding to the core SOX21-AS1 promoter. Samples from at least three independent immunoprecipitations were analyzed. 
Tissue sections were following a deparaffinized and rehydrated process, and then block endogenous peroxidase activity by being treated with 3\% H2O2 in methanol for 30 min to. Tissue sections were then rinsed twice for five minutes in phosphate-buffered saline (PBS) and blocked with $10 \%$ normal goat serum. After resin, the samples were incubated with a primary anti-YAP1 (\#4912, Cell Signaling, MA), anti-YAP1 (s127) (\#13008, Cell Signaling, MA). Sections were incubated with secondary antibodies and stained with DAB according to the manufacturer's protocol, mounted on slides, and photographed using a digital microscope camera (Nikon, Tokyo, Japan).

For multiple color staining, we processed the slide strictly according to the instruction of Opal 4- Anti-Rabbit Automation IHC kit (NEL830001KT, AKOYA science) with primary antibody to anti-AXL (\#8661, Cell Signaling, MA), Anti-HPV16 E6 + HPV18 E6 antibody (ab70, Abcam, Cambridge) and Anti-Human Papillomavirus 16 (E7) antibody (ab30731, Abcam, Cambridge) at $4{ }^{\circ} \mathrm{C}$ overnight, and photographed using a digital microscope camera (Nikon, Tokyo, Japan).

\section{Western-blot}

For western blotting, proteins were extracted from cultured cells using RIPA buffer containing phenylmethanesulfonylfluoride (PMSF) (Beyotime, Nantong, China). An equal amount of proteins $(100 \mu \mathrm{g})$ was separated by $7.5 \% / 12.5 \%$ sodium dodecyl sulfate-polyacrylamide gel electrophoresis (SDS-PAGE) and transferred to a polyvinylidene fluoride (PVDF) membrane. Primary polyclonal antibodies targeting anti-YAP1 (\#4912, Cell Signaling, MA), anti-YAP1 (s127) (\#13008, Cell Signaling, MA), LATS1 (\#9153, Cell Signaling, MA), 14-3-3 (\#8312, Cell Signaling, MA), Anti- HPV16 E6 + HPV18 E6 antibody (ab70, Abcam, Cambridge) and Anti-Human Papillomavirus 16 (E7) antibody (ab30731, Abcam, Cambridge) and TAZ (ab652, Abcam, Cambridge) were purchased from Abcam (Cambridge, MA, USA). The secondary antibodies used were anti-rabbit HRP-linked (Abcam). The blots were developed using ECL reagent (Millipore). An equal amount of protein loading in each lane was confirmed using the $\beta$-actin antibody. ImageJ software was used to quantify the integrated density of the band.

\section{Cell Counting Kit-8 (CCK-8) assay and transwell cell migration}

For the migration and invasion assays, cells were placed into the upper chamber $(8-\mu \mathrm{m}$ pore size; Millipore), which was coated with (invasion assay) or without (migration assay) the matrigel mix (BD Biosciences, San Jose, CA, USA) according to the manufacturer's protocol. After incubation for $24 \mathrm{~h}$, the cells located on the upper membrane of the trans-well chambers were removed with cotton wool, while cells that had migrated or invaded through the membrane were 
fixed with methanol for $10 \mathrm{~min}$, followed by staining with $0.1 \%$ crystal violet for $30 \mathrm{~min}$ at $37^{\circ} \mathrm{C}$. Three randomly selected microscopic fields were counted per field for each group. Experiments were independently repeated three times.

\section{Luciferase reporter assay}

The promoter region of SOX21-AS1 containing the wild-type or mutant potential target site for TEADs was designed and synthesized in Genescript Co. (Nanjing, China) and inserted into the pGL4 Vector (Promega, WI). For luciferase assay, C33A was transfected with pGL4-SOX21-AS1 WT pro or pGL4-SOX21-AS1 MU pro, with pCMV-E6, pCMV-E7 or control (GenePharma, China) using Lipofectamine 2000 (Thermofisher, CA). LPA treated Cells were harvested $48 \mathrm{~h}$ after transfection for luciferase assay using a Dual-Luciferase ${ }^{\circledR}$ Reporter Assay System (Promega, WI) according to the manufacturer's protocol.

\section{Statistical analysis}

Data are presented as mean \pm SD. $\chi^{2}$ tests and the Student's t-test analysis of variances were used to evaluate statistical differences in demographic and clinical characteristics. All expression experiments conducted in vitro were repeated at least three times with triplicate samples. Pearson's correlation analysis was used to analyze the relationship between associated factors. Statistical analysis was performed using STATA 9.2 and presented with GraphPad Prism software (CA, USA). In all cases, $\mathrm{P}<0.05$ was considered significant.

\section{Results}

\section{SOX21-AS1 is an HPV16 infection-specific IncRNA in human CCs}

To explore human HPV16 infection-specific lncRNAs, we screened the human cervical cancer RNA sequencing (RNA-seq) data from The Cancer Genome Atlas (TCGA) database. According to the report by Tang et al.[15], we obtained 18 samples of other HPV infection and 55 samples of HPV 16 infection within TGCA_CESC database. After statistical analysis( $t$-test), we found 873 lncRNAs significantly differently expressed between HPV 16 and other HPV infection including 186 down-regulated and 687 up-regulated lncRNAs (Fig. 1 a-c). Among the top 20 most significantly up-regulated lncRNAs, we found that only SOX21-AS1(ENSG00000227640) and HCP5 (ENSG00000206337) were significantly up-regulated in tumor compared to normal tissues based on searching a on-line TCGA analyzing tool Gepia (Fig.1d). Gene Set Enrichment Analysis (GSEA) were 
also carried out on SOX21-AS1 and HCP5 in TCGA-CESC, interestingly, we found that high expression of SOX21-AS1 was positively correlated to the Hippo signaling pathway (Fig. 1e and f). SOX21-AS1 is a long non-coding RNA located in human chromosome 13 with Ensembl ID: ENSG00000227640.

\section{SOX21-AS1 is an E6 protein-associated IncRNA}

As HPV16 has two vital oncogenic proteins, E6 and E7[5], we evaluated AXL, the downstream traget gene for YAP1 activation[16], E6, and E7 protein expression levels in CCs tissues using multi-color staining. The $40 \mathrm{CCs}$ patients were further categorized into E6+E7+ (9 cases), E6+E7- (6 cases), E6-E7+ (5 cases), and E6-E7- (20 cases) groups (Fig. 2a). SOX21-AS1 showed a significant positive correlation with AXL protein expression level (Fig. 2b). We divided 40 patients with CCs based on SOX21-AS1 expression (Fig. 2c) and found that SOX21-AS1 level was significantly related to E6 but not E7 (Fig. 2d and e).

\section{E6-associated SOX21-AS1 is closely related to the expression and nuclear localization of}

\section{YAP1}

We investigated the relationship between HPV16 infection and activation of YAP1 protein in hCCs by immunohistochemistry (IHC) and found that YAP1 expression in E6+ group (E6+E7- and E6+E7+) was significantly higher than that in E6- group (E6-E7- and E6-E7+) (Fig. 3a). The nuclear localization of YAP1 in E6+ group was much higher than that in E6- group (Fig. 3a). As the nuclear localization of YAP1 is closely related to its activation[16], these results suggest that E6 expression may be related to the activation of YAP1. To confirm this observation, we detected YAP1(s127), YAP1 degradation-related phosphorylation residue, and found it to be much lower in E6+ CCs than in E6- group (Fig. 3b). We also analyzed the correlation between nuclear YAP1 or YAP1(s127) and SOX21-AS1 in hCCs. The results showed that the expression of SOX21-AS1 positively correlated with nuclear YAP-1 but negatively correlated with YAP1(s127) (Fig. 3c-e).

A previous study has shown that E6 protein can promote YAP1/TAZ transnational activity by binding to the PDZ domain of TAZ[17]. We found that E6 level was positively related to SOX21-AS1. We investigated the potential binding site of TEADs, co-transcriptional factors for YAP1/TAZ, in the 
promoter region of SOX21-AS1 and detected one potential binding site (Fig. 3f). We mutated this binding site and found a significant decrease in the promoter activity as compared with the WT promoter following treatment with E6, LPA (YAP-1 activator), E6 + LPA, or E7 + LPA. E7 protein alone had no apparent effect on the promoter activity of SOX21-AS1 (Fig. 3g). To further confirm that TEADs can bind to the promoter region of SOX21-AS1, we performed a ChIP assay using antibody to TEAD1-4 and p65, we found that TEAD1-4 can bind to the promoter region of SOX21-AS1 specifically (Fig. 3h).

We performed a co-immunoprecipitation (co-IP) assay with SiHa and SiHa-E6/E7 (SiHa cells overexpression E6 and E7) cells using TAZ antibody, and found that E6, but not E7, could bind to TAZ (Fig. 3i). These results imply that the enhanced expression of SOX21-AS1 in hCCs was attributed to the increase in the transcriptional activity of YAP1/TAZ/TEADs promoted by E6. We speculate that SOX21-AS1 may serve as an E6-specific lncRNA.

\section{SOX21-AS1 enhances the proliferation and invasion of hCCs cells by YAP1 activation}

To investigate the biological functions of SOX21-AS1, we studied the expression of SOX21-AS1 in different hCCs cell lines, including C33A, ME180 (HPV16-), SiHa, and CaSki (HPV16+) cells. We found that C33A and ME180 exhibited very low E6 and lnc-HPV16 expression levels, while SiHa and CaSki cells has significantly higher expression of E6 and lnc-HPV, particularly CaSki cells owing to its high copy of HPV16 (Fig. 4a) [18].

We found that the proliferation and invasion abilities of C33A and ME180 cells were significantly lower than those of CaSki and SiHa cells (Fig. 4b,c). We overexpressed SOX21-AS1 in C33A cells (C33A-SOX21-AS1) and knocked down its expression in CaSki cells (CaSki-lnc-SOX21-AS1 short-hairpin RNA [shRNA]) (Fig. 4d). As a result, the proliferation and invasion abilities were significantly increased in C33A-SOX21-AS1 cells but decreased in CaSki-SOX21-AS1 shRNA cells (Fig. 4e,f). We also found that CA3, a specific inhibitor of YAP1/TAZ, significantly decreased the proliferation and invasion of C33A-SOX21-AS1 and CaSki cells (Fig. 4g,h).

We also investigated the activation of YAP1 by western blot analysis, and found that the level of total YAP1 was the highest and that of YAP1(s127) was the lowest in CaSki cells (Fig. 4i). 
Furthermore, we activated YAP1 by lysophosphatidic acid (LPA) $(0.1 \mathrm{uM})$, and found a decrease in YAP(s127) level and an increase in total YAP1 level in all cell lines, especially CaSki cells (Fig. 4j). Total YAP1 activation also increased in C33A-SOX21-AS1 cells but decreased in Caki-SOX21-AS1 shRNA cells with or without treatment with LPA (Fig. 4k and 1). Therefore, these results suggest that SOX21-AS1 promoted the proliferation and invasion of hCCs cells via YAP1 activation.

\section{SOX21-AS1 can stabilize YAP-1 by binding to YAP1(s127)}

We used the online tool catRAPID to predict the possibility of binding between SOX21-AS1 and YAP1. The results suggested that SOX21-AS1 (201-252nt) has the most potential to directly bind to YAP1 protein at the region of 102-154 amino acids (Fig. 5a and b). Next, we found that the sense probe, but not the anti-sense probe, of SOX21-AS1 could pull-down YAP-1 (Fig. 5c). To verify the specific binding site of SOX21-AS1 on YAP1, we constructed a mutated form (201-252 deletion) of SOX21-AS1 (SOX21-AS1 MU) and found that the 201-252 fragment in SOX21-AS1 were essential for its binding to YAP1 (Fig. 5d).

To investigate the effect of SOX21-AS1 on YAP1 nuclear translocation, we performed immunofluorescence (IF) staining and found that the overexpression of SOX21-AS1, but not SOX21-AS1 MU, in C33A and CaSki cells could enhance YAP1 nuclear translocation (Fig. 5e). Next, we investigated the effect of YAP-1 and SOX21-AS1 combination on the promotion of cell proliferation and invasion and found that SOX21-AS1 MU overexpression could significantly attenuate the positive effects of SOX21-AS1 on cell proliferation and invasion (Fig. $5 \mathrm{f}$ and $\mathrm{g}$ ).

\section{Discussion}

In present study, we found a lncRNA named SOX21-AS1 that could enhance the nuclear translocation of YAP1 by binding to 101-125 amino acids, which carry an important phosphorylation residue of YAP1 (s127). The s127 residue of YAP1 could cause proteasome-induced degradation by 14-3-3-derived ubiquitination [16]. The overexpression of SOX21-AS1 blocked the binding between 14-3-3 and YAP1 by facilitating its binding to s127 region, thereby reducing protein degradation. YAP1 is a core protein of the Hippo signaling pathway which is an essential and highly conserved 
pathway in cell proliferation, apoptosis, and organ development[19]. YAP1 itself has no transcriptional activity, it is a transcriptional co-activator when combined with its TAZ and TEADs [19]. All of these three components are indispensable for downstream transcripts boost, therefore, the nuclear translocation of YAP1 is the key regulatory manner, and this process is blocked by Hippo pathway when it is in the“on"state[20, 21]. The"on"state of the Hippo pathway is considered as tumor suppressive functions, since amounts of researches revealed its"on" state is capable of inhibiting cell proliferation, promoting apoptosis and regulating stem/progenitor cell expansion[20, 21].

We also found that the positive effects on cell proliferation and invasion of SOX21-AS1 were attributed to YAP1 activation, and that the YAP/TAZ activation inhibitor CA3 could completely attenuate the carcinogenic effects of SOX21-AS1. Several other lncRNAs have been reported to exert tumorigenic effects by targeting Hippo signaling. Li et al. reported that the crosstalk between ROR1-HER3 and Hippo pathway promotes breast cancer bone metastasis [22]. Zhu et al. also reported that lncBRM associated with BRM to initiate the BRG1/BRM switch and that the BRG1-embedded BAF complex could trigger the activation of YAP1 signaling [23].

We investigated the expression of SOX21-AS1 in hCCs and found that the transcription of SOX21-AS1 was associated with HPV16 infection and driven by E6 following binding to TAZ. Similar results were also reported by other research groups [12]. The E6 protein is an essential nuclear and cytoplasmic protein of about $18 \mathrm{kDa}$ [4]. It contains four cysteine arrays that constitute two relatively large zinc fingers, both of which are required for full function: 1. a transcriptional trans-activator, or coactivator[4]; 2. E6 modulates normal cellular processes by binding to cellular proteins, in particular, E6AP and E6BP[24]; 3. E6 targets the degradation of p53[24]; and 4. E6 of high-risk HPVs induces immortalization of primary cell cultures, and E6 of low-risk HPVs augments the ability of E7 to immortalize cells [24]. We found that SOX21-AS1 is one of the downstream productions of E6/TAZ/TEADs complex in HPV16 infected human CCs cells. Similar results were also reported by other research groups. Thus, SOX21-AS1 was driven by E6/YAP1/TAZ/TEADs and could promote proliferation and invasion of hCCs cells by preventing YAP1 degradation by the E3 
ubiquitination ligase 14-3-3. We believe that the high expression of SOX21-AS1 is an important cause for hCCs and that SOX21-AS1 could serve as a potential therapeutic marker for hCCs.

In summary, exploring the TCGA database, we discovered an HPV16 infection-related lncRNA, SOX21-AS1 in human CCs. High expression of SOX21-AS1 was seriously related to TMN stage and HPV E6 protein expression. The mechanistic study revealed that SOX21-AS1 was driven by E6/YAP1/TAZ/TEADs, it can promote cell proliferation and invasion in CCs cells by binding to YAP1 in the region containing s127, preventing YAP1 degradation by E3 ubiquitination ligase 14-3-3. We think that high expression of SOX21-AS1 is an important cause and potential therapeutic target for human CCs.

\section{Declarations:}

\section{Authors' contributions}

Conception and design: JRQ and YYL. Collection and assembly of data: JJW, FH, SX, and YXS Data analysis and interpretation: JJW and FH Contribution of reagents, materials, and analysis tools wrote the paper: JJW, FH and QHZ. All authors read and approved the final manuscript.

\section{Consent for publication}

All authors consent for publication.

\section{Conflict of Interest}

The authors declared no competing interests.

\section{Availability of data and materials}

Not applicable

\section{Funding}

This work was supported by grants from the National Natural Science Foundation (Grant Number: 81572370,81772569 and 81972266 to R.J.).

\section{Ethics approval and consent to participate}

Animal experiments were approved and performed in accordance with the institutional guidelines for animal care of animal ethics committee of the 1st People's Hospital of Kunshan (KSL-2017018). All institutional and national guidelines for the care and use of laboratory animals were followed.Informed consent to be included in the study, or the equivalent, was obtained from all patients.

\section{Acknowledgments}

Not applicable 


\section{Reference}

1. Ferris, R.L., et al., Effect of Adding Motolimod to Standard Combination Chemotherapy and Cetuximab Treatment of Patients With Squamous Cell Carcinoma of the Head and Neck: The Active8 Randomized Clinical Trial. JAMA Oncol, 2018. 4(11): p. 1583-1588.

2. Fakhry, C., et al., Human Papillomavirus Testing in Head and Neck Carcinomas: ASCO Clinical Practice Guideline Endorsement of the College of American Pathologists Guideline. J Clin Oncol, 2018. 36(31): p. 3152-3161.

3. $\quad$ Guido, R., Cervical Cancer Screening. Clin Obstet Gynecol, 2018. 61(1): p. 40-51.

4. Parmin, N.A., et al., Human Papillomavirus E6 biosensing: Current progression on early detection strategies for cervical Cancer. Int J Biol Macromol, 2019. 126: p. 877-890.

5. Yeo-Teh, N.S.L., Y. Ito, and S. Jha, High-Risk Human Papillomaviral Oncogenes E6 and E7 Target Key Cellular Pathways to Achieve Oncogenesis. Int J Mol Sci, 2018. 19(6).

6. Mirabello, L., et al., HPV16 E7 Genetic Conservation Is Critical to Carcinogenesis. Cell, 2017. 170(6): p. 1164-1174 e6.

7. Kranjec, C., et al., Modulation of basal cell fate during productive and transforming HPV-16 infection is mediated by progressive E6-driven depletion of Notch. J Pathol, 2017. 242(4): p. 448-462.

8. Mahata, S., et al., Berberine modulates AP-1 activity to suppress HPV transcription and downstream signaling to induce growth arrest and apoptosis in cervical cancer cells. Mol Cancer, 2011. 10: p. 39.

9. Liu, Y., et al., An AP-1 binding site mutation in HPV-16 LCR enhances E6/E7 promoter activity in human oral epithelial cells. Virus Genes, 2002. 24(1): p. 29-37.

10. Shai, A., et al., The human papillomavirus E6 oncogene dysregulates the cell cycle and contributes to cervical carcinogenesis through two independent activities. Cancer Res, 2007. 67(4): p. 1626-35.

11. Sanchez-Perez, A.M., et al., Disruption of the human papillomavirus type 16 E2 gene protects cervical carcinoma cells from E2F-induced apoptosis. J Gen Virol, 1997. 78 ( Pt 11): p. 3009-18.

12. Mori, S., et al., Human Papillomavirus 16 E6 Upregulates APOBEC3B via the TEAD Transcription Factor. J Virol, 2017. 91(6).

13. Barr, J.A., et al., Long non-coding RNA FAM83H-AS1 is regulated by human papillomavirus 16 E6 independently of 53 in cervical cancer cells. Sci Rep, 2019. 9(1): p. 3662.

14. Villota, C., et al., Expression of mitochondrial non-coding RNAs (ncRNAs) is modulated by high risk human papillomavirus (HPV) oncogenes. J Biol Chem, 2012. 287(25): p. 21303-15.

15. Tang, K.W., et al., The landscape of viral expression and host gene fusion and adaptation in human cancer. Nat Commun, 2013. 4: p. 2513.

16. Totaro, A., T. Panciera, and S. Piccolo, YAP/TAZ upstream signals and downstream responses. Nat Cell Biol, 2018. 20(8): p. 888-899.

17. Webb Strickland, S., et al., Human Papillomavirus E6 interaction with cellular PDZ domain proteins modulates YAP nuclear localization. Virology, 2018. 516: p. 127-138.

18. Ahn, W.S., et al., A major constituent of green tea, EGCG, inhibits the growth of a human cervical cancer cell line, CaSki cells, through apoptosis, G(1) arrest, and regulation of gene expression. DNA Cell Biol, 2003. 22(3): p. 217-24.

19. Shibata, M., K. Ham, and M.O. Hoque, A time for YAP1: Tumorigenesis, immunosuppression and targeted therapy. Int J Cancer, 2018. 143(9): p. 2133-2144.

20. Hillmer, R.E. and B.A. Link, The Roles of Hippo Signaling Transducers Yap and Taz in Chromatin Remodeling. Cells, 2019. 8(5).

21. Kim, C.L., S.H. Choi, and J.S. Mo, Role of the Hippo Pathway in Fibrosis and Cancer. Cells, 2019. 8(5).

22. Li, C., et al., A ROR1-HER3-IncRNA signalling axis modulates the Hippo-YAP pathway to regulate bone metastasis. Nat Cell Biol, 2017. 19(2): p. 106-119.

23. Zhu, P., et al., LncBRM initiates YAP1 signalling activation to drive self-renewal of liver cancer stem cells. Nat Commun, 2016. 7: p. 13608.

24. Mills, A.M., et al., HPV E6/E7 mRNA In Situ Hybridization in the Diagnosis of Cervical Low-grade Squamous Intraepithelial Lesions (LSIL). Am J Surg Pathol, 2018. 42(2): p. 192-200. 


\section{Figure legend}
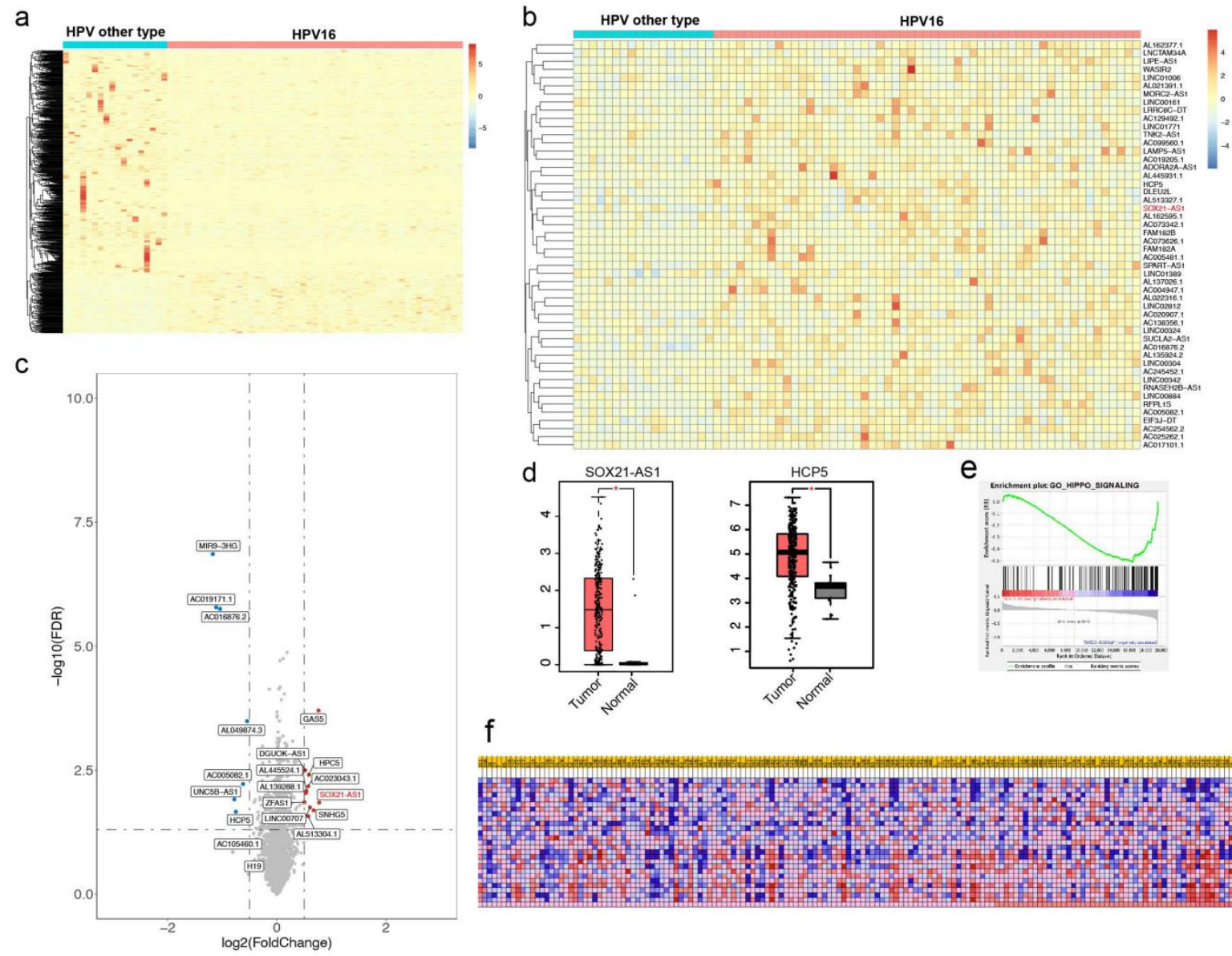

e

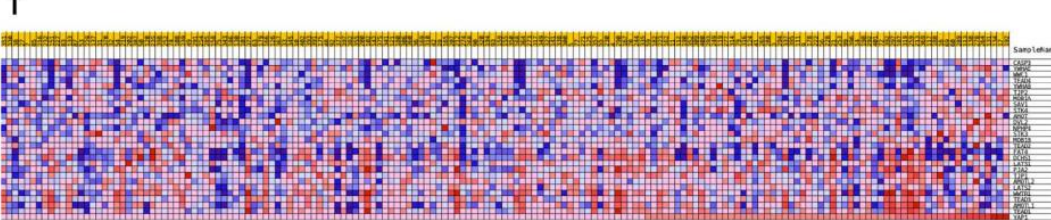

Figure 1. SOX21-AS1 specifically overexpressed in human HPV 16 infected human cervical squamous cell carcinoma.

a and b: Heatmap figures of differential expressed and top 30 down-regulated lnc-RNAs expression compared between HPV16 positive and other HPV (HPV 18 exclusive) in human cervical squamous cell carcinoma (hCCs) tissues from the database of The Cancer Genome Atlas (TCGA). c: A volcano map showing top up-regulated and down-regulated differential expressed lncRNAs were presented. d:The expression of SOX21-AS1 and HCP5 were compared between tumor and normal tissues with CSEA dataset in TCGA. e and f: A Gene sent Enrichment Analysis (GSEA) targeting high and low expression of SOX21-AS1 showed that the expression of SOX21-AS1 was seriously related to Hippo Signaling Pathway. 


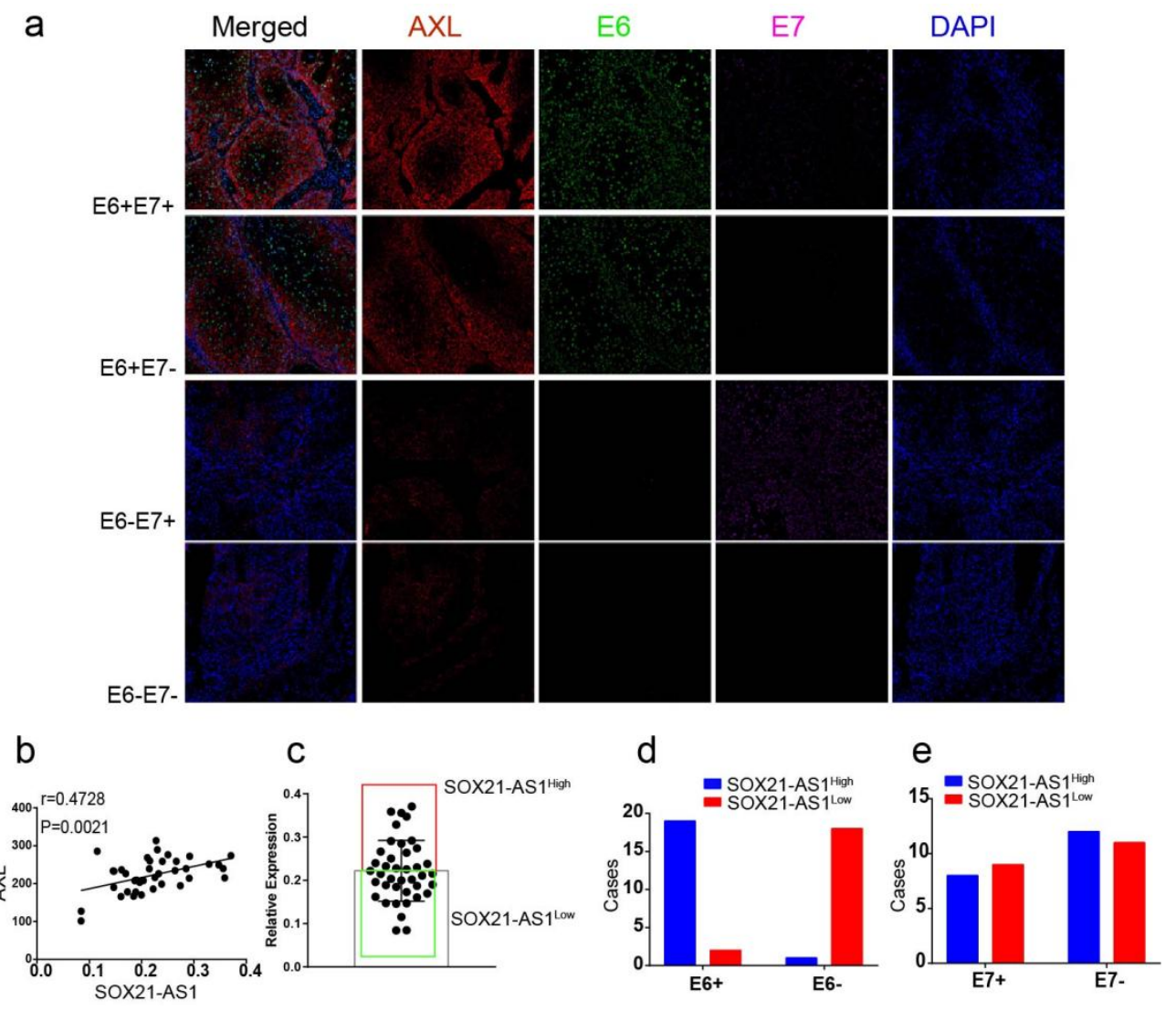

Figure 2. SOX21-AS1 is an E6 protein associated IncRNA.

a. Representative figures for multiple-color staining for AXL, E6 and E7 expression in human CCs samples sub- categorized as indicated in the figure $(\times 100)$. b: Linear correlation analysis between transcription of SOX21-AS1 and AXL protein expression Integrated Optical Density Value (IOD) in human CCs tissues. The figures were analyzed by using image-J. c: The selected human CCs tissues were sub-divided into Lnc-HPV16 high $(n=20)$ and low $(n=20)$ expression group according to the Median. $d$ and e: The relation between SOX21-AS1 expression and E6 or E7 expression in human CCs were analyzed by chi-square test. Data represent means \pm SEM of at least three independent experiments. $* \mathrm{P}<0.05, * * \mathrm{P}<0.01$. 
a

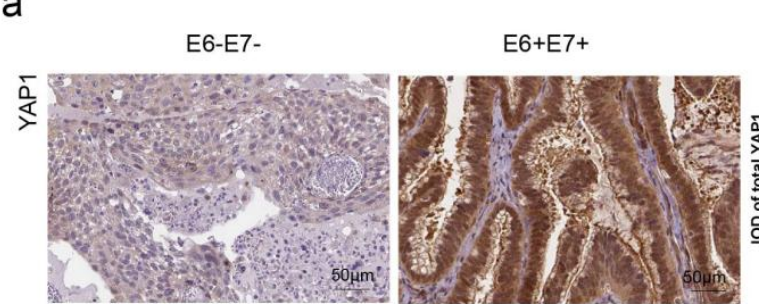

E6-E7+

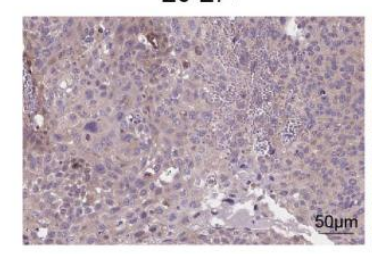

b
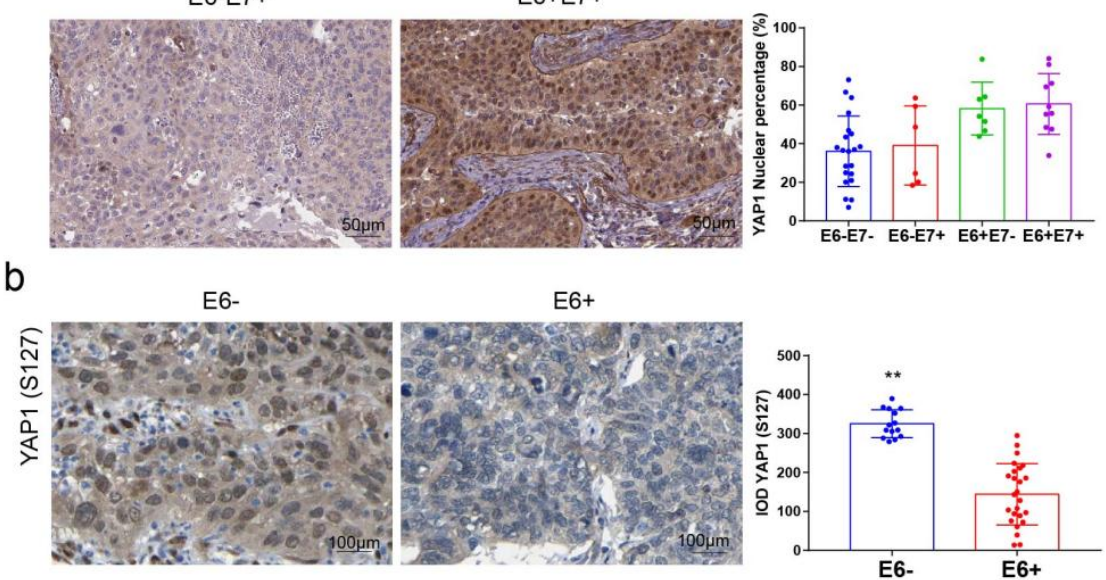
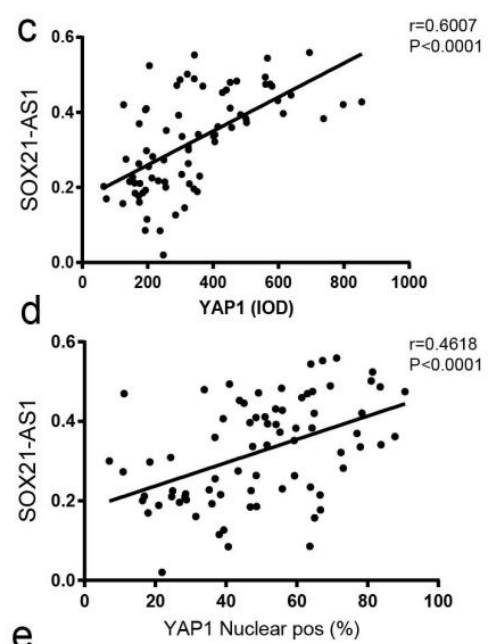

e

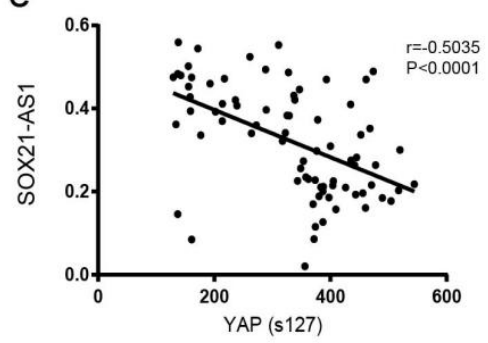

f

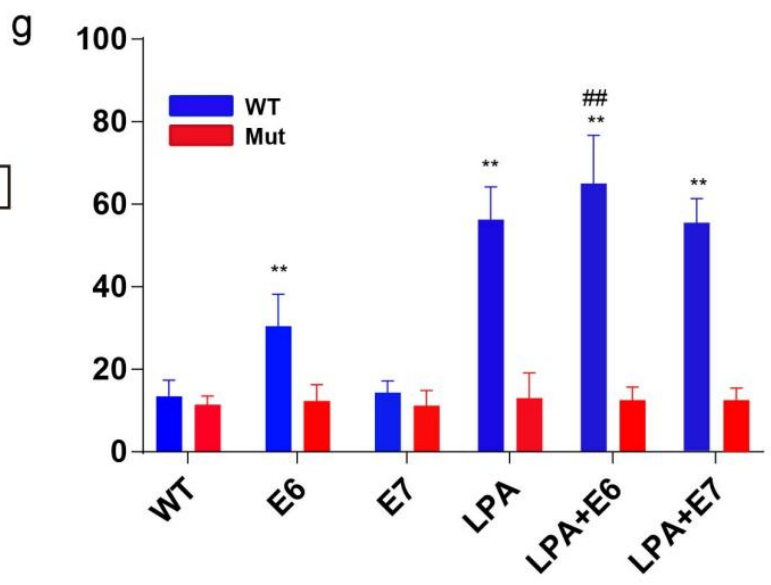

h
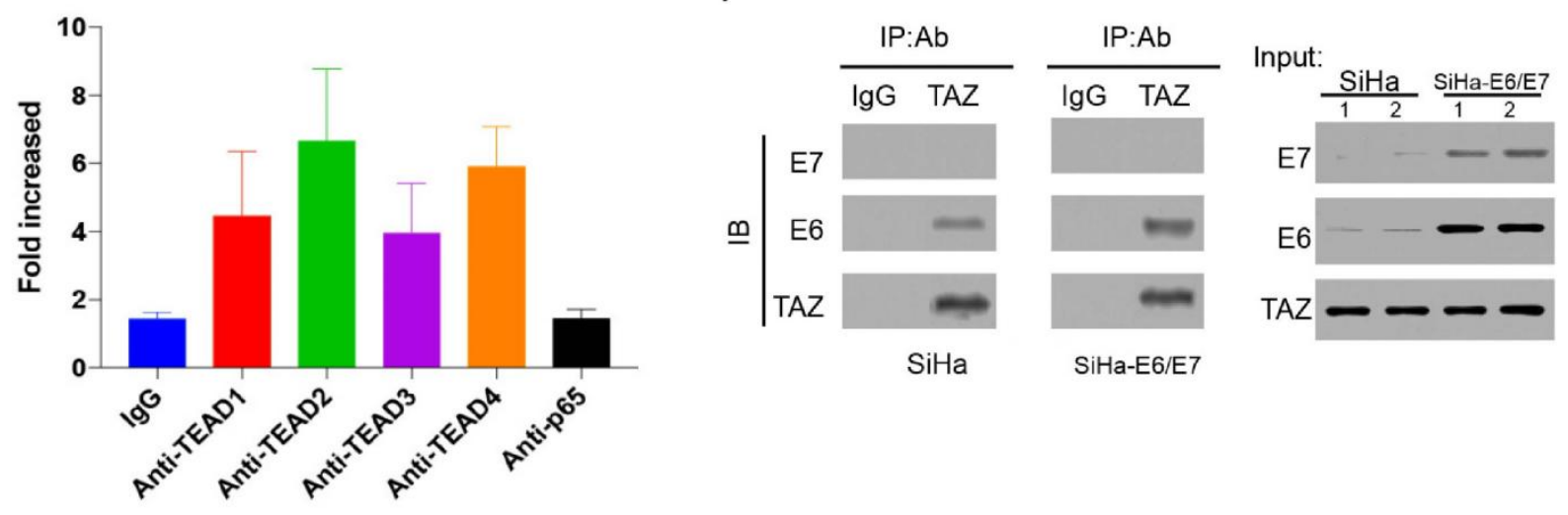

Figure 3: E6 associated SOX21-AS1 is closely related to the expression and nuclear localization 


\section{of YAP1}

$a$ and $b$ : Representative figures and analysis of immunohistochemistry staining for YAP1 $(\times 100)$ and YAP1(s127) $(\times 200)$ in human CCs sub-grouped indicated in the figure. The figures were analyzed by using image-J. c-e: The linear correlation analysis between expression of SOX21-AS1 and YAP1 expression (IOD value) or percentage of YAP1 nuclear translocation and YAP1 (s127) in human CCs were presented. f: Sketch of potential TEADs binding site and mutation design within SOX21-AS1 promoter region. g: Luciferase reporter assay of E6, E7 and LPA treatment on the activity of Wide Type (WT) and TEADs binding site mutation (Mut) SOX21-AS1 promoter. h: Chromatin immunoprecipetation (ChIP) assay was performed by using antibody to TEAD 1-4, p65 and IgG serve as a negative control. i: co- IP assay study the binding between TAZ and E6 or E7, the protein complex was immuno- precipitated with TAZ antibody and further detected by E6 or E7 antibody. Data represent means \pm SEM of at least three independent experiments. ${ }^{*} \mathrm{P}<0.05, * * \mathrm{P}<$ 0.01 . 

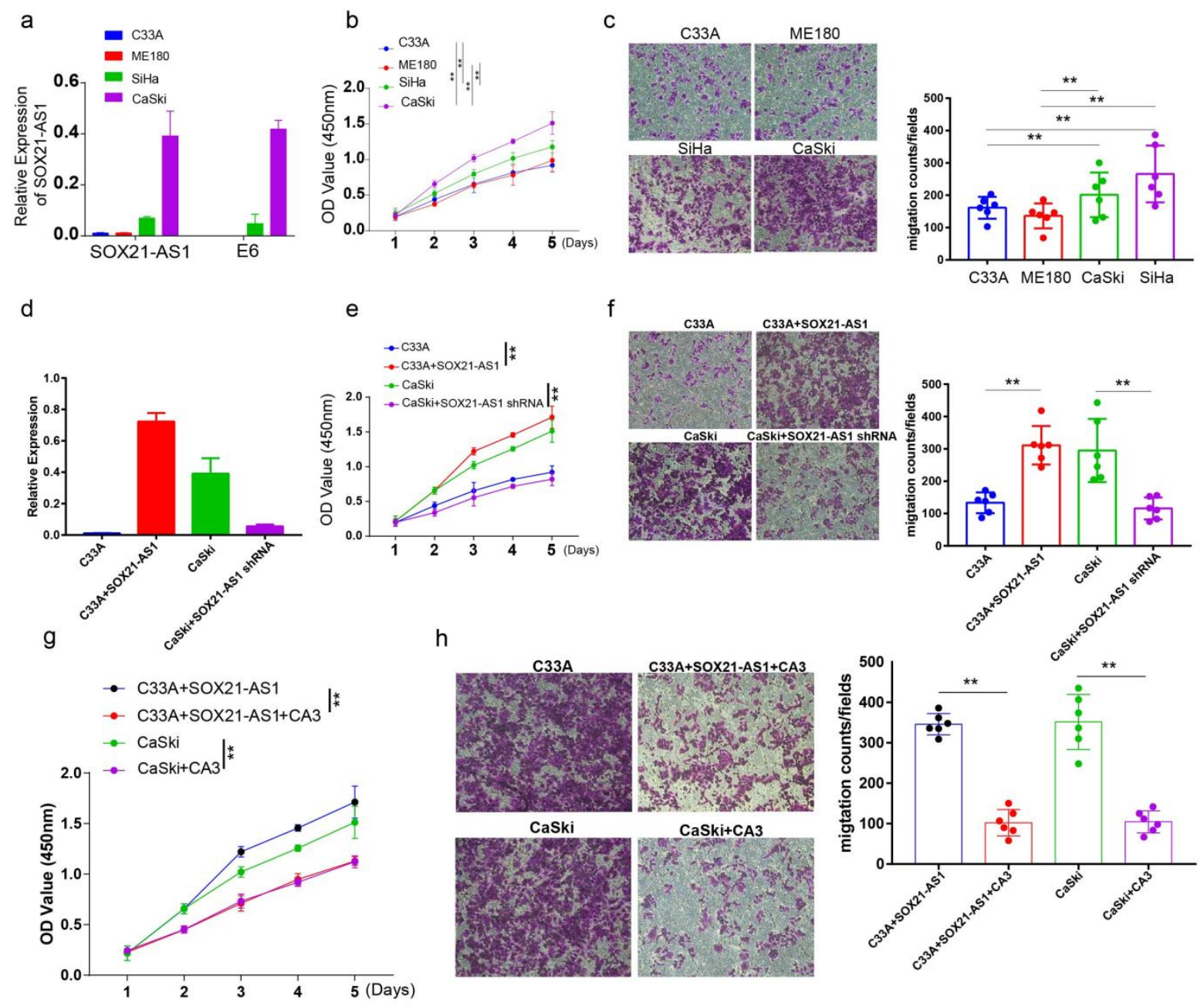

i
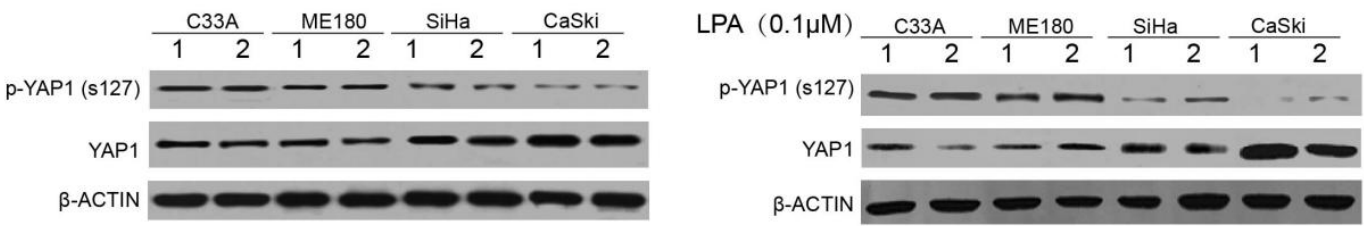

$\mathrm{k}$

।
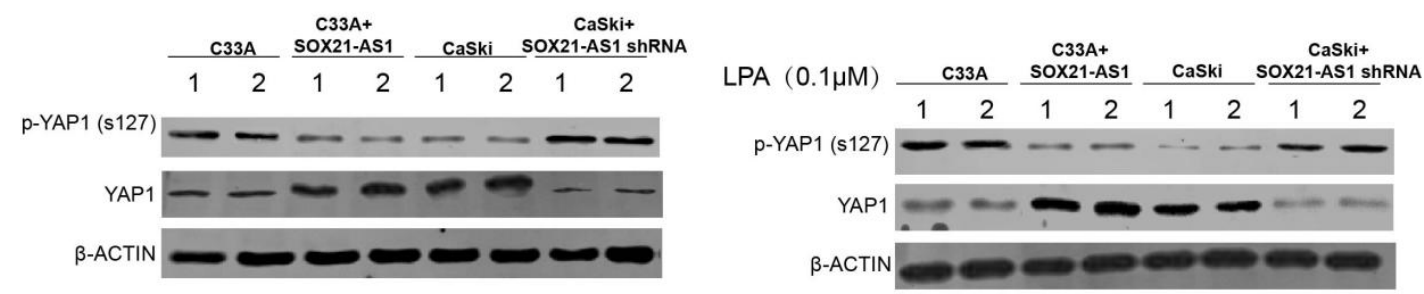

Figure 4: SOX21-AS1 can enhance the proliferation and invasion of human cervical squamous cell carcinoma cells by YAP1 activation.

a: The expression of SOX21-AS1 was detected by real-time PCR in C33A, ME180, SiHa, and CaSki cells. b: The cell proliferation of C33A, ME180, SiHa, and CaSki cells were detected by using 
CCK8 assay. c: The cell invasion ability of C33A, ME180, SiHa, and CaSki cells were detected by using transwell. The expression of SOX21-AS1, cell proliferation and cell invasion of C33A, C33A-SOX21-AS1, CaSki and CaSki-SOX21-AS1 shRNA were presented in d, e and f. g and h: The cell proliferation and cell invasion of C33A-SOX21-AS1 and CaSki treated with or without CA3 were analyzed by CCK 8 and transwell based cell invasion assay. i-j: The hCCs cell line were treated with or without LPA $(0.1 \mu \mathrm{M})$, protein expression of YAP1(s127), YAP1 controlled by $\beta$-ACTIN were detected by using western-blot. Data represent means \pm SEM of at least three independent experiments. $* \mathrm{P}<0.05, * * \mathrm{P}<0.01$. 
a

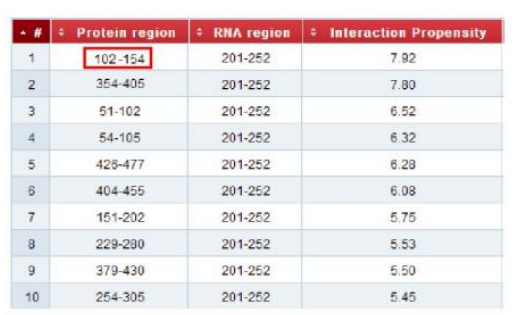

c

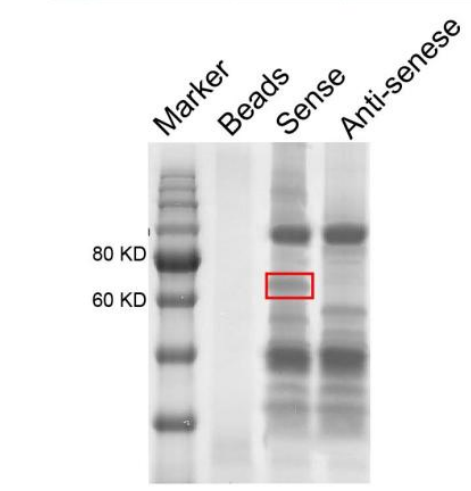

pull-down:YAP 1

Input YAP1

e

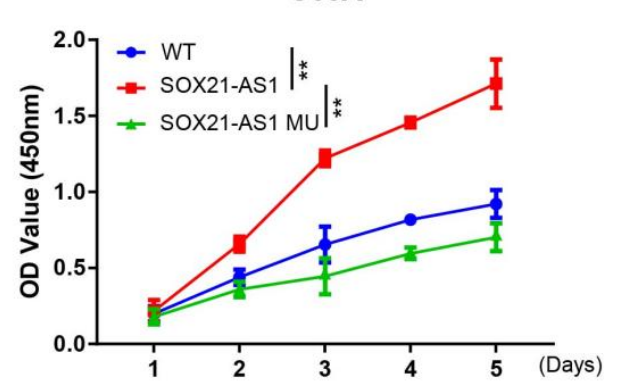

f
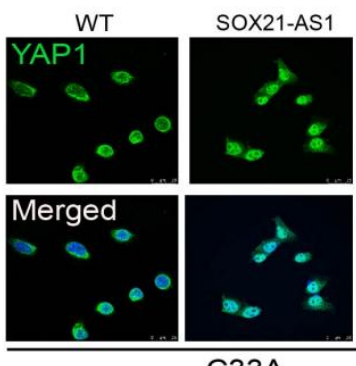

g

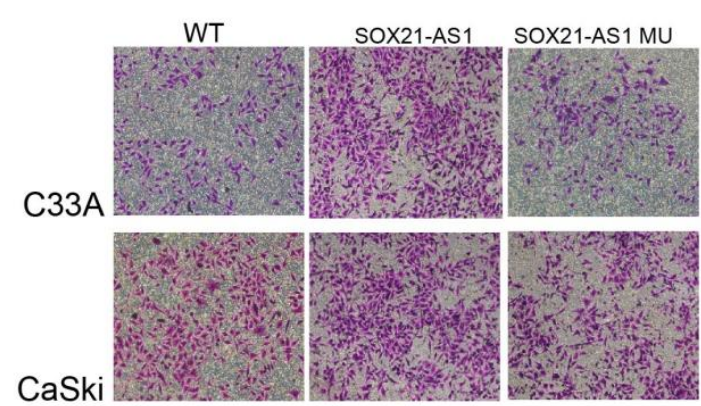

b
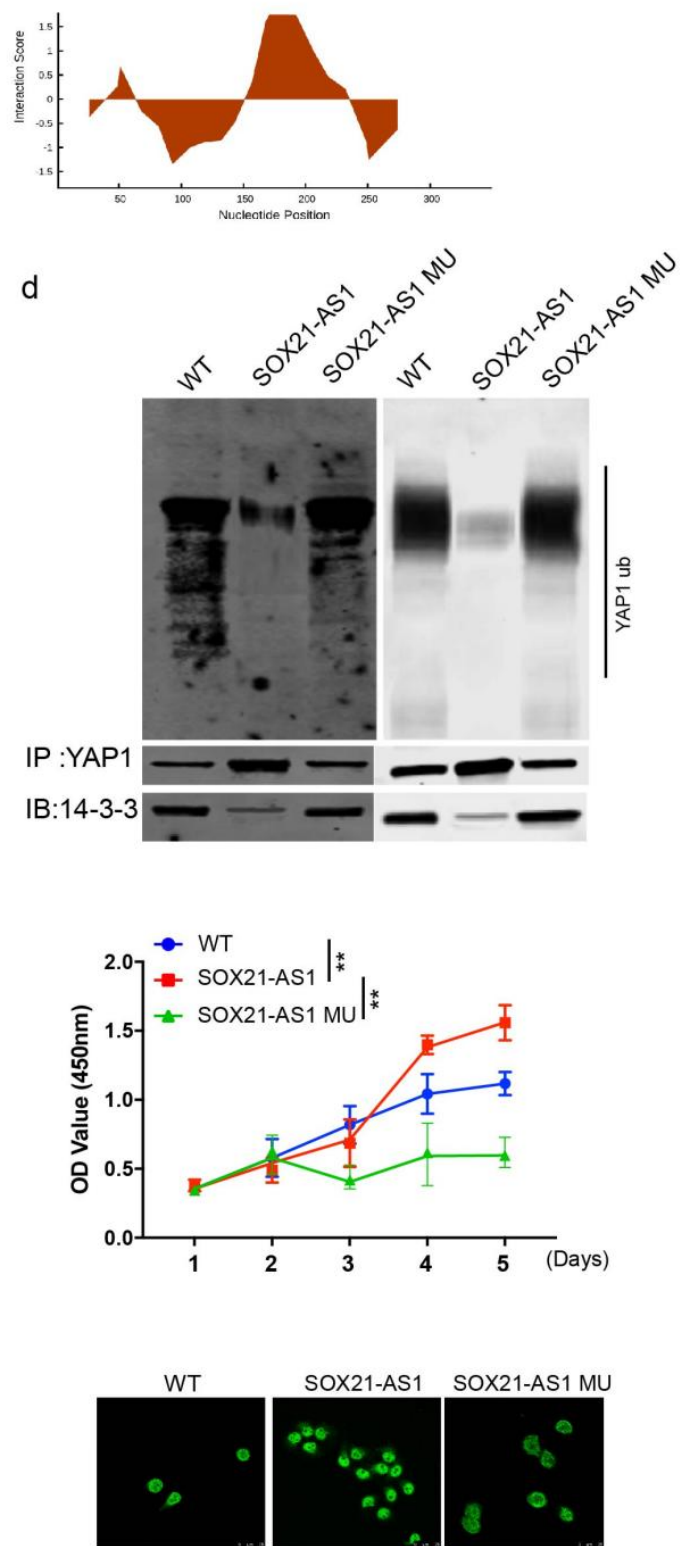

SOX21-AS1 SOX21-AS1 MU
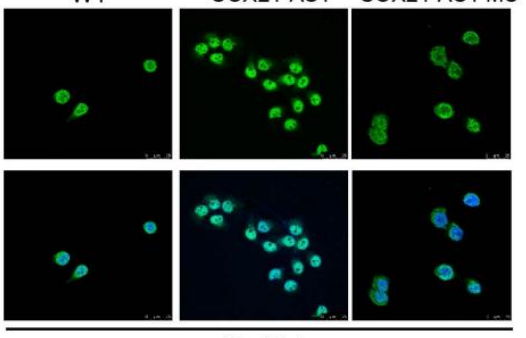

CaSki

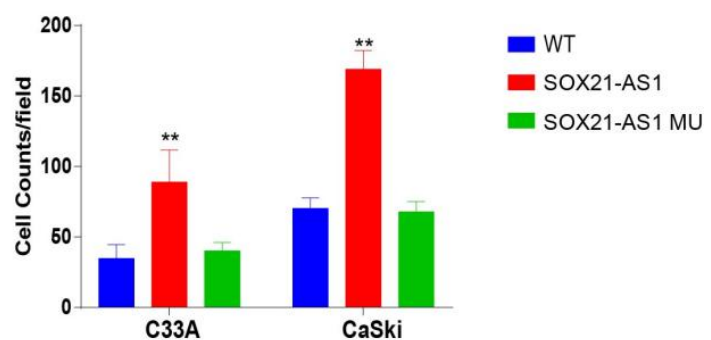

Figure 5. SOX21-AS1 can stabilize YAP-1 protein and activate the downstream pathway by binding to YAP1 (s127) residue.

$a, b$ The potential binding sites between SOX21-AS1 and YAP1 were predicted by catRAPID, the 
possible binding region and interaction score were presented. c. RNA pull-down using sense and anti-sense probe specific for SOX21-AS1 and controlled by beads. The precipitated and input total protein was detected by YAP1 Antibody using western-blot. d. Ubiquitination detection of YAP1 in C33A and CaSki cells transfected by WT and MU SOX21-AS1. e: Cell proliferation of C33A and CaSki cells transfected by WT and MU SOX21-AS1. f: YAP1 nuclear-translocation in C33A and CaSki cells transfected by WT and MU SOX21-AS1 were detected by using IF staining assay. g: Cell invasion of C33A and CaSki cells transfected by WT and MU SOX21-AS1 were accessed by using transwell cell migration assay. Data represent means \pm SEM of at least three independent experiments. $* \mathrm{P}<0.05, * * \mathrm{P}<0.01$. 


\section{Figures}

a

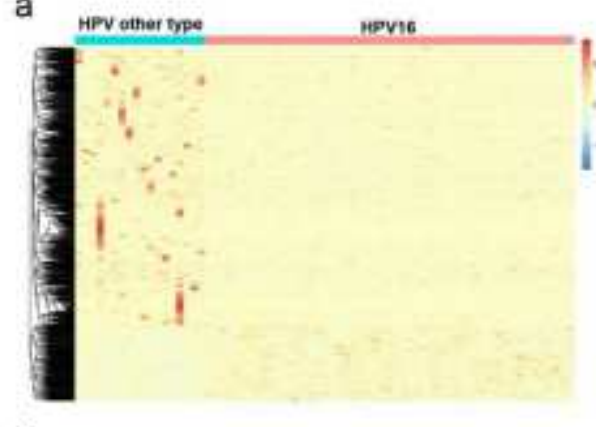

c

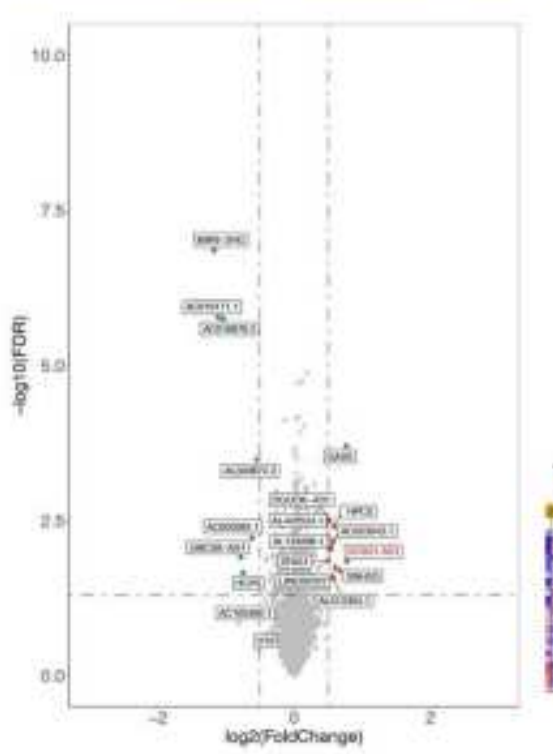

b hPvother ove

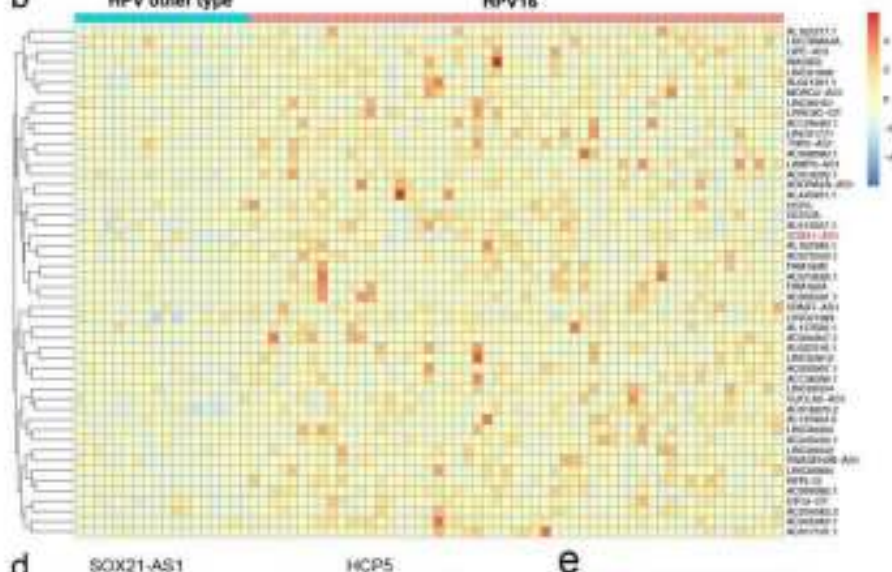

d
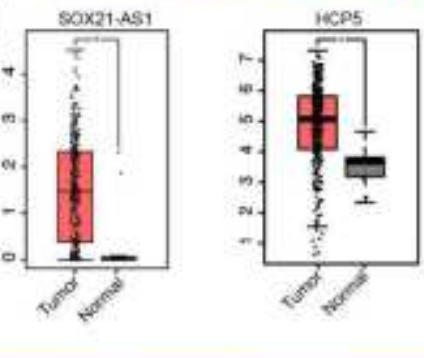

e

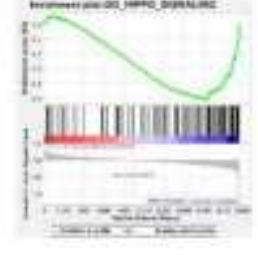

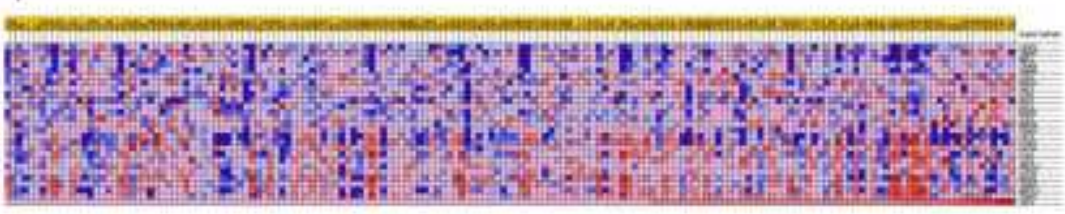

Figure 1

SOX21-AS1 specifically overexpressed in human HPV 16 infected human cervical squamous cell carcinoma. a and b囚Heatmap figures of differential expressed and top 30 down-regulated Inc-RNAs expression compared between HPV16 positive and other HPV (HPV 18 exclusive) in human cervical squamous cell carcinoma (hCCs) tissues from the database of The Cancer Genome Atlas (TCGA). c: A volcano map showing top up-regulated and down-regulated differential expressed IncRNAs were presented. d:The expression of SOX21-AS1 and HCP5 were compared between tumor and normal tissues with CSEA dataset in TCGA. e and f: A Gene sent Enrichment Analysis (GSEA) targeting high and low expression of SOX21-AS1 showed that the expression of SOX21-AS1 was seriously related to Hippo Signaling Pathway. 


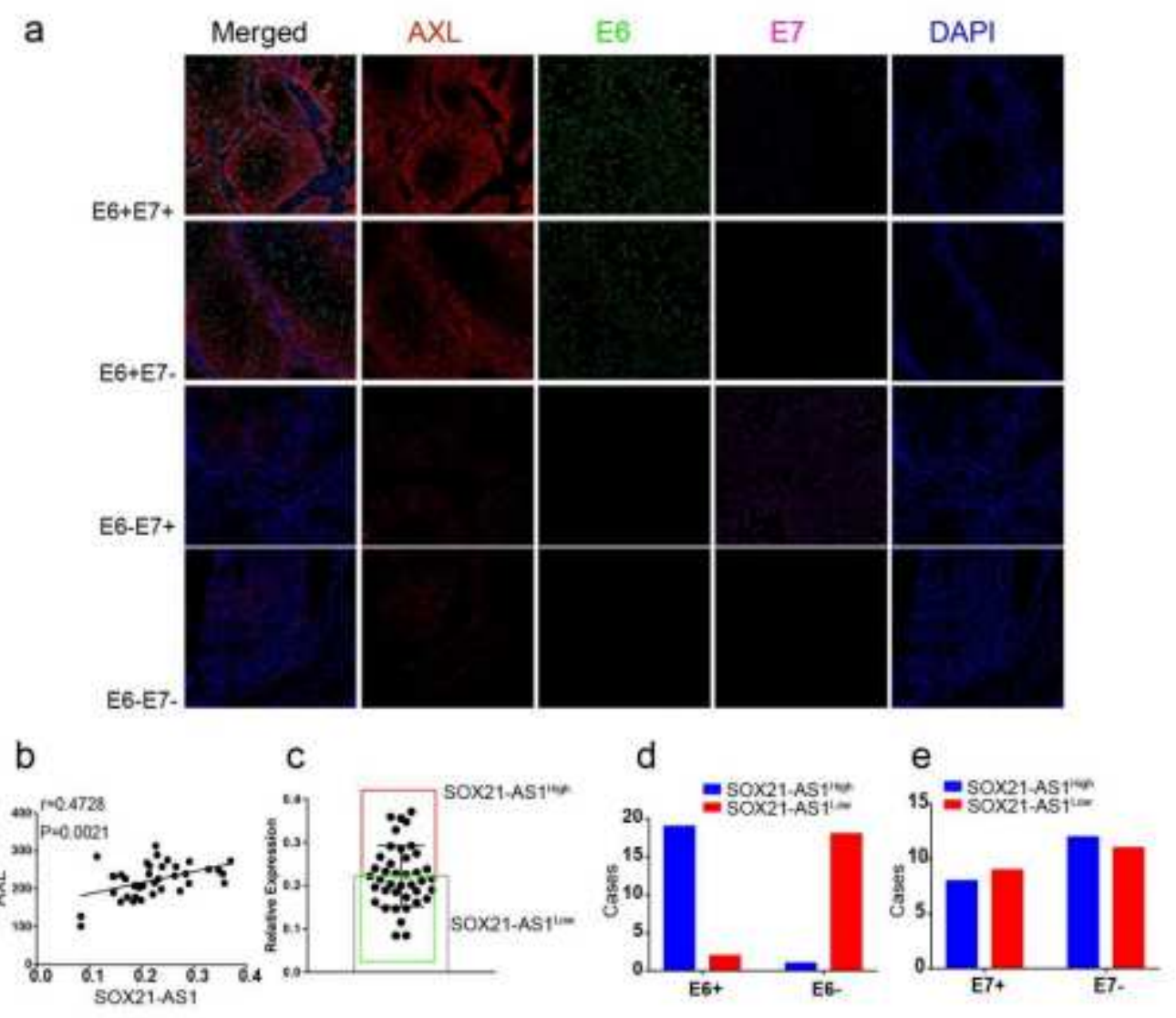

\section{Figure 2}

SOX21-AS1 is an E6 protein associated IncRNA. a. Representative figures for multiple-color staining for $A X L, E 6$ and E7 expression in human CCs samples sub- categorized as indicated in the figure $(\times 100)$. b: Linear correlation analysis between transcription of SOX21-AS1 and AXL protein expression Integrated Optical Density Value (IOD) in human CCs tissues. The figures were analyzed by using image-J. c: The selected human CCs tissues were sub-divided into Lnc-HPV16 high $(n=20)$ and low $(n=20)$ expression group according to the Median. $d$ and e: The relation between SOX21-AS1 expression and E6 or E7 expression in human CCs were analyzed by chi-square test. Data represent means \pm SEM of at least three independent experiments. ${ }^{\star} \mathrm{P}<0.05,{ }^{\star} * \mathrm{P}<0.01$. 
a
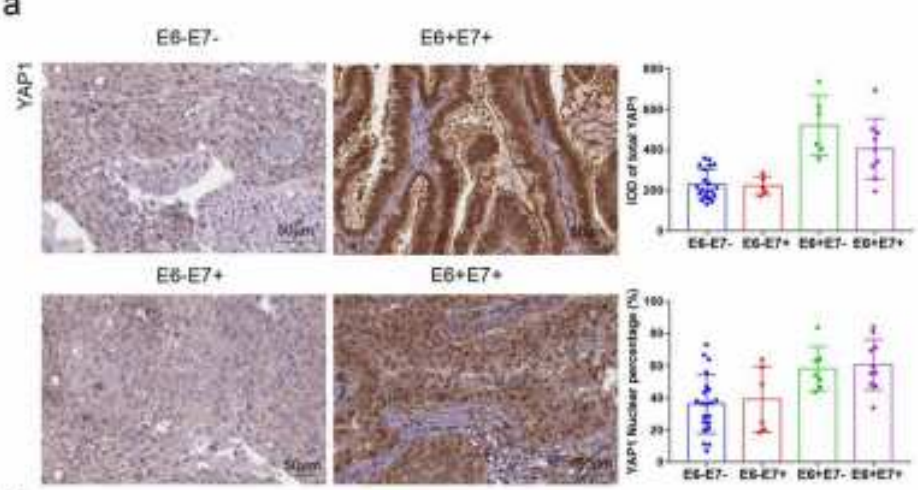

b
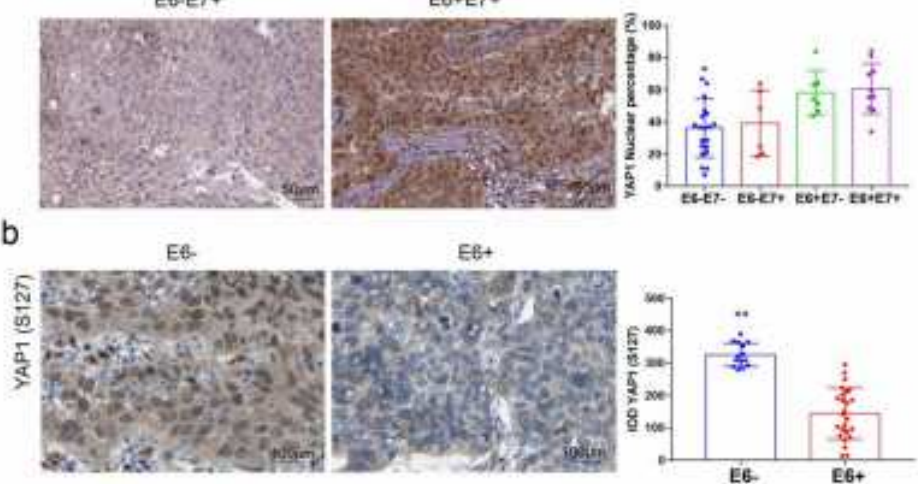

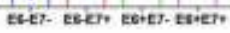

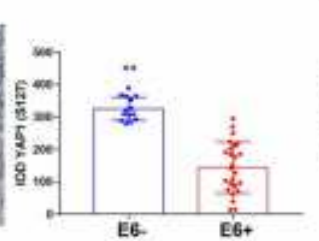

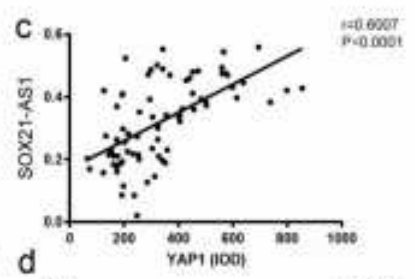
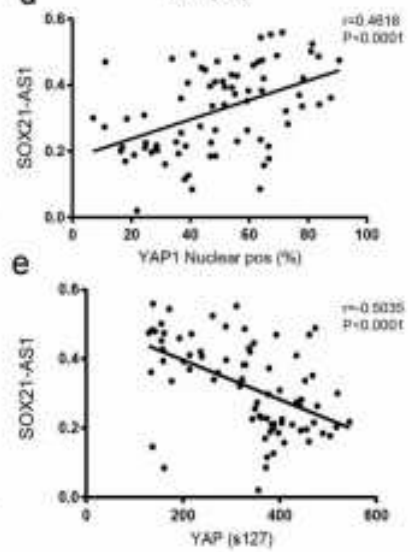

f

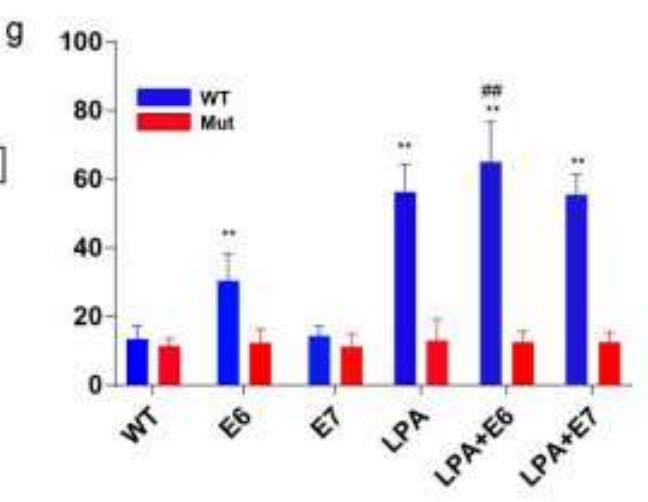

$\mathrm{h}$

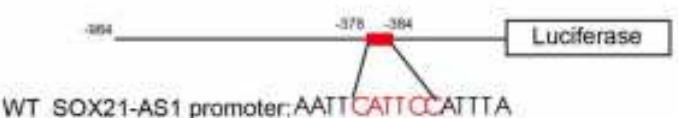

Mut SOX21-AS1 promoter: AATT AITTA

i
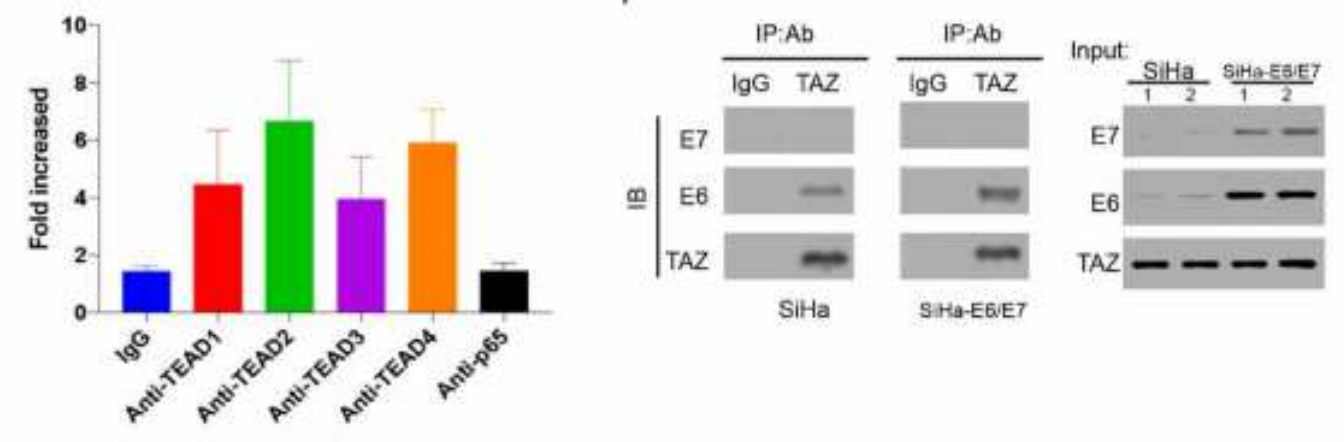

\section{Figure 3}

E6 associated SOX21-AS1 is closely related to the expression and nuclear localization of YAP1 a and b: Representative figures and analysis of immunohistochemistry staining for YAP1 ( $\times 100)$ and YAP1(s127) $(\times 200)$ in human CCs sub-grouped indicated in the figure. The figures were analyzed by using image-J. Ce: The linear correlation analysis between expression of SOX21-AS1 and YAP1 expression (IOD value) or percentage of YAP1 nuclear translocation and YAP1 (s127) in human CCs were presented. f: Sketch of 
potential TEADs binding site and mutation design within SOX21-AS1 promoter region. g: Luciferase reporter assay of E6, E7 and LPA treatment on the activity of Wide Type (WT) and TEADs binding site mutation (Mut) SOX21-AS1 promoter. h: Chromatin immunoprecipetation (ChIP) assay was performed by using antibody to TEAD 1-4, p65 and IgG serve as a negative control. i: co- IP assay study the binding between TAZ and E6 or E7, the protein complex was immuno- precipitated with TAZ antibody and further detected by $\mathrm{E} 6$ or $\mathrm{E7}$ antibody. Data represent means \pm SEM of at least three independent experiments. ${ }^{*} \mathrm{P}$ $<0.05, * \star P<0.01$.
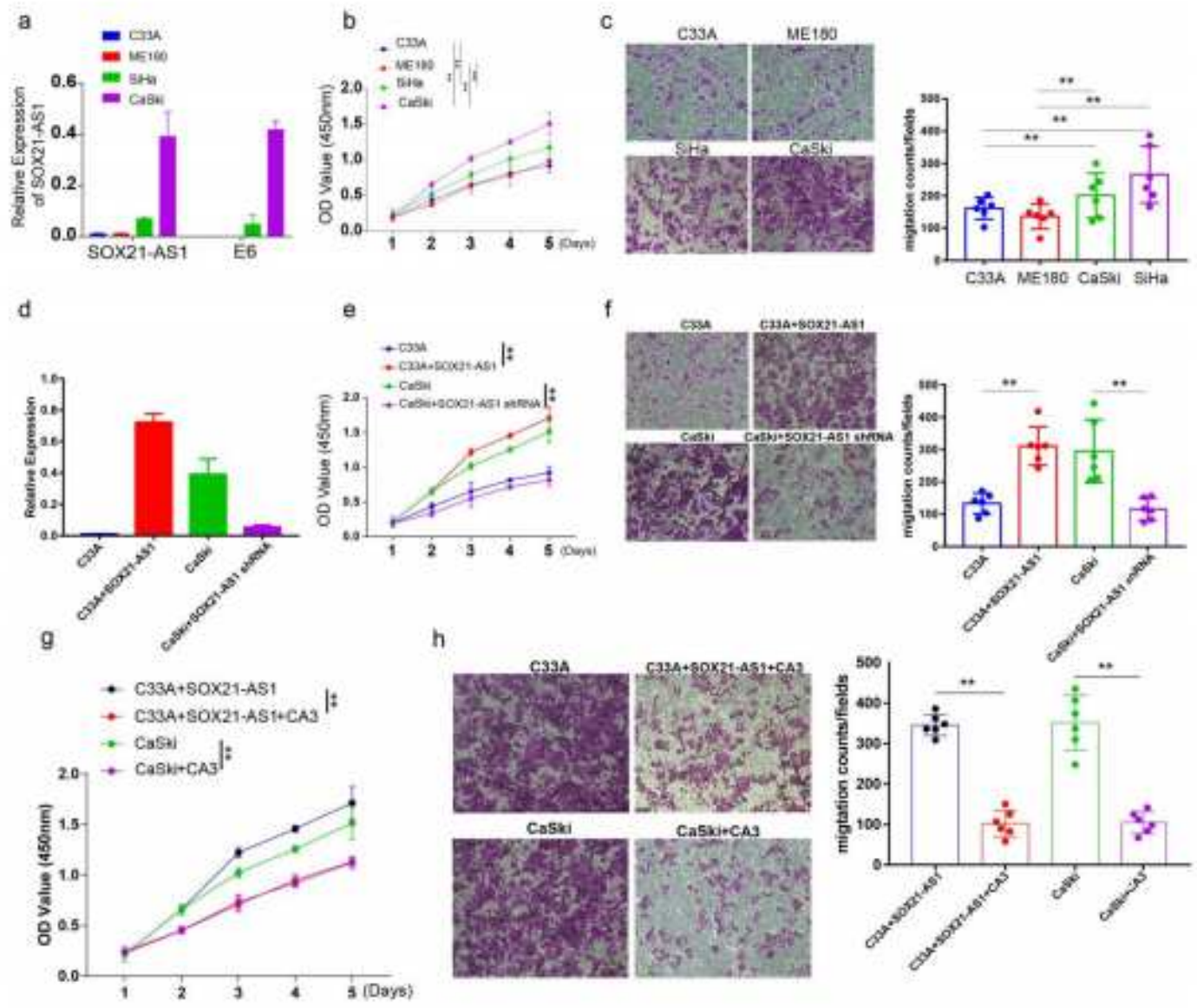

i
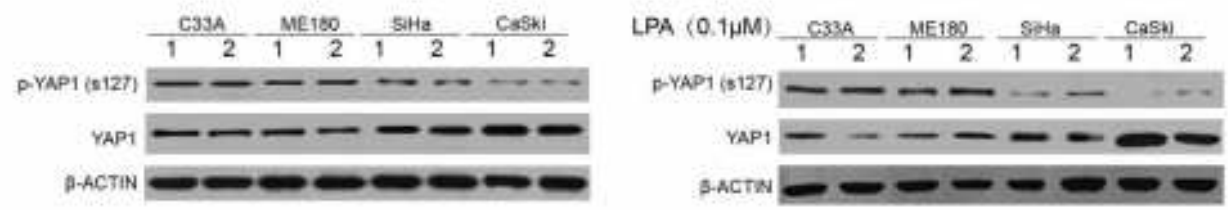

k.

I
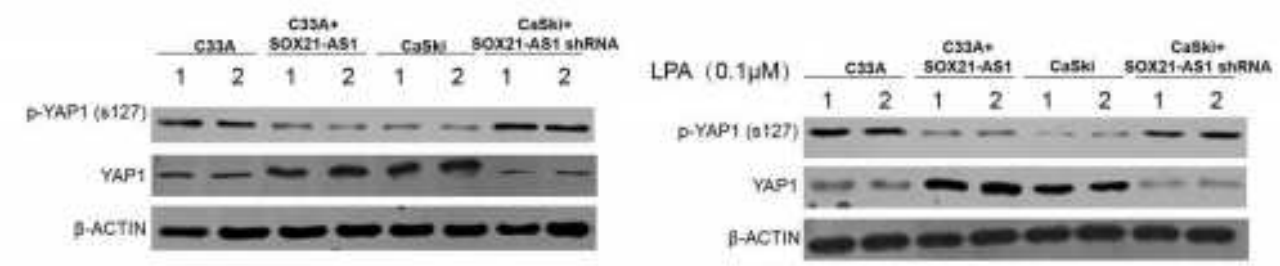


\section{Figure 4}

SOX21-AS1 can enhance the proliferation and invasion of human cervical squamous cell carcinoma cells by YAP1 activation. a: The expression of SOX21-AS1 was detected by real-time PCR in C33A, ME180, $\mathrm{SiHa}$, and CaSki cells. b: The cell proliferation of C33A, ME180, SiHa, and CaSki cells were detected by using CCK8 assay. C: The cell invasion ability of C33A, ME180, SiHa, and CaSki cells were detected by using transwell. The expression of SOX21-AS1, cell proliferation and cell invasion of C33A, C33A-SOX21AS1, CaSki and CaSki-SOX21-AS1 shRNA were presented in $\mathrm{d}$, e and $\mathrm{f} . \mathrm{g}$ and $\mathrm{h}$ : The cell proliferation and cell invasion of C33A-SOX21-AS1 and CaSki treated with or without CA3 were analyzed by CCK8 and transwell based cell invasion assay. i-j: The hCCs cell line were treated with or without LPA $(0.1 \mu \mathrm{M})$, protein expression of YAP1(s127), YAP1 controlled by $\beta$-ACTIN were detected by using western-blot. Data represent means \pm SEM of at least three independent experiments. ${ }^{*} \mathrm{P}<0.05,{ }^{*} \mathrm{P}<0.01$. 
a

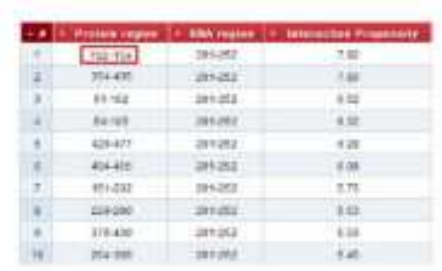

$\mathrm{c}$

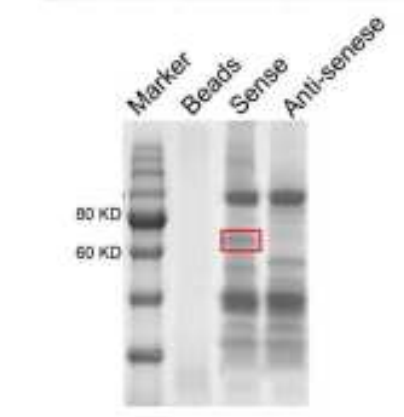

pull-down YAP 1 Input YAPt

e

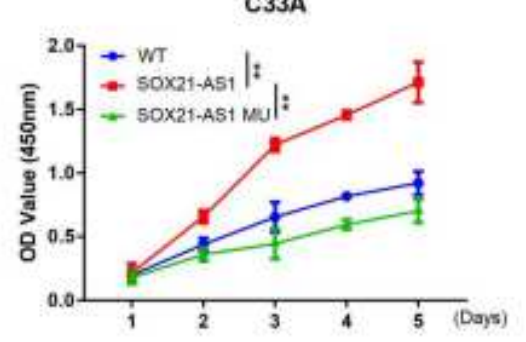

$f$
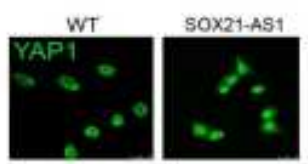

Merged
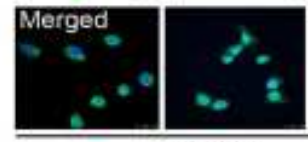

C33A

g

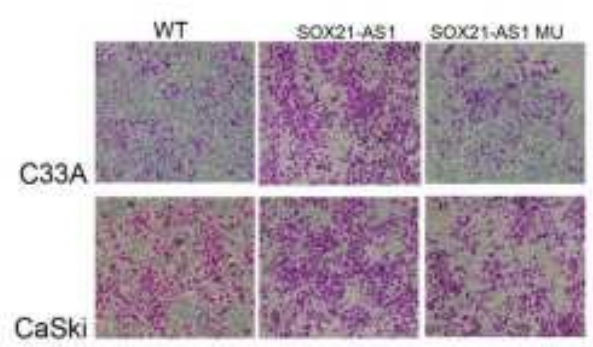

b
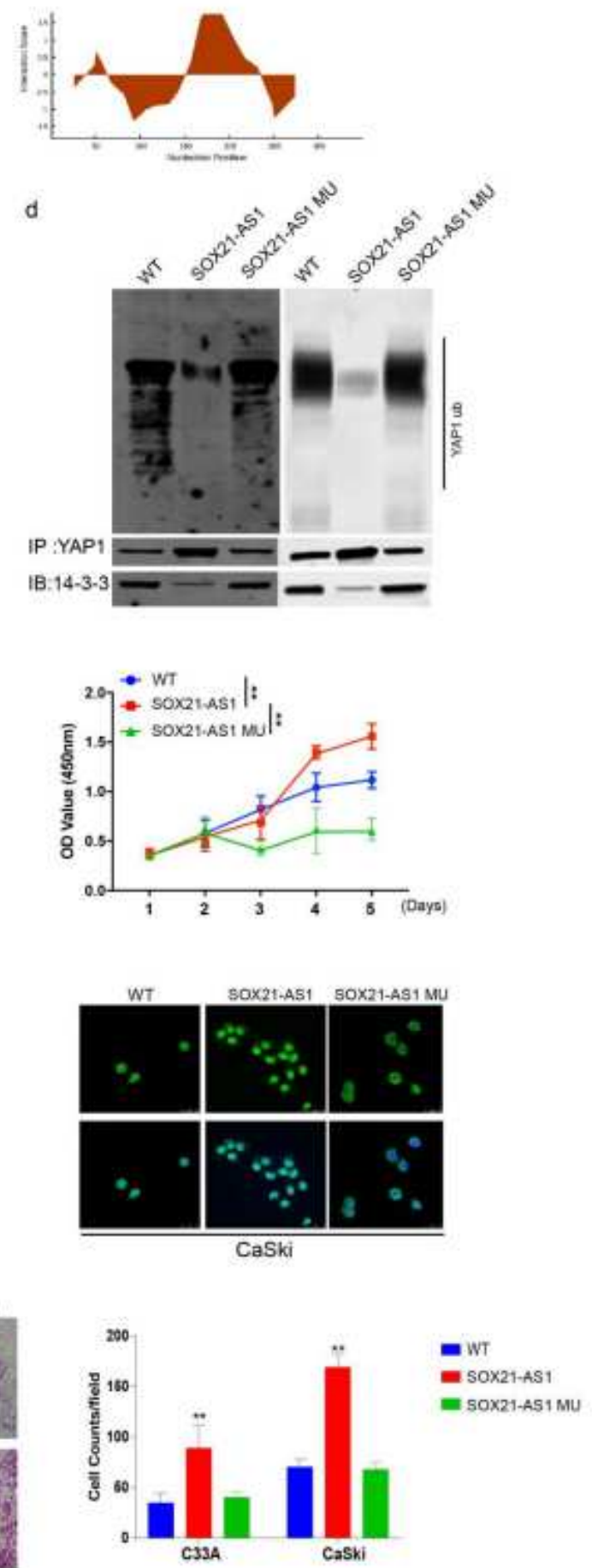

\section{Figure 5}

SOX21-AS1 can stabilize YAP-1 protein and activate the downstream pathway by binding to YAP1 (s127) residue. a,b The potential binding sites between SOX21-AS1 and YAP1 were predicted by catRAPID, the possible binding region and interaction score were presented. c. RNA pull-down using sense and antisense probe specific for SOX21-AS1 and controlled by beads. The precipitated and input total protein was detected by YAP1 Antibody using western-blot. d. Ubiquitination detection of YAP1 in C33A and CaSki 
cells transfected by WT and MU SOX21-AS1. e: Cell proliferation of C33A and CaSki cells transfected by WT and MU SOX21-AS1. f: YAP1 nuclear-translocation in C33A and CaSki cells transfected by WT and MU SOX21-AS1 were detected by using IF staining assay. g: Cell invasion of C33A and CaSki cells transfected by WT and MU SOX21-AS1 were accessed by using transwell cell migration assay. Data represent means \pm SEM of at least three independent experiments. ${ }^{*} P<0.05,{ }^{*} \mathrm{P}<0.01$. 